\title{
Preparation of a Novel Organic-Inorganic Hybrid Material for Highly Efficient Degradation of Methyl Orange
}

\author{
Yue Wang ${ }^{1, a^{*}}$ and Jian Sheng Guo ${ }^{2, b}$ \\ ${ }^{1,2}$ Key Laboratory of Textile Science and Technology, Ministry of Education, College of Textiles, \\ Donghua University, 2999 North Renmin Road, Shanghai 201620, P. R. China \\ a1109001@mail.dhu.edu.cn, ${ }^{\mathrm{b}}$ jsguo@dhu.edu.cn
}

Keywords: Ionic liquid; Methyl orange; Degradation; Palladium; Catalyst

\begin{abstract}
Porous silica was first synthesized using sodium silicate as silicon source. Ionic liquid-polymer functionalized porous silica was prepared via the chain transfer polymerization of grafting an ionic liquid monomer, 1-allyl-3-methylimidazolium bromide, onto the surface of the silica . The resultants were characterized by scanning electron microscope, nitrogen physisorption experiment, thermogravimetric analysis, Fourier transform infrared spectra and so on. The novel hybrid material loaded with Pd can be used as a catalyst. The experimental results indicate that the catalyst has a prominent effect on the degradation of methyl orange in the presence of hydrogen peroxide. The reasonable explanation is that the metal component of the catalyst stimulated the dissociation of hydrogen peroxide to release abundant highly active free radicals which can rapidly ruin the structure of methyl orange molecules in water. Finally, a probable catalytic mechanism based on diffusion was proposed.
\end{abstract}

\section{Introduction}

Methyl orange is a heavy pollutant known to be carcinogenic and mutagenic, widely existing in various wastewater from textile, dyeing, leather and plastics industries. For the purposes of environmental accountability and favorable health, the decomposition of methyl orange has been paid much attention within the last two decades. Advanced oxidation processes are highly efficient and novel methods for the decomposition of methyl orange. Some oxidants such as hydrogen peroxide have the ability to disrupt methyl orange molecules but the oxidant is usually stable in the presence of methyl orange. In order to enhance the rate of the degradation of methyl orange, a few auxiliary measures is necessary. The inorganic or organic components in some compounds have been proved to be efficient activators for hydrogen peroxide to produce various free radicals and intermediates[1-3]. $\mathrm{Xu}$ et al found that bicarbonate anion can stimulate the dissociation of hydrogen peroxide giving active oxygen species, which disrupt methyl orange molecules[2]. Ember et al studied the mechanism of $\mathrm{Mn}$ ( II ) activating hydrogen peroxide in the presence of bicarbonate anion and pointed that the active species formed in the reactions such as the $\mathrm{Mn}(\mathrm{IV})=\mathrm{O}$ intermediate and $\cdot \mathrm{OH}$ radicals are the motivation of the degradation of methyl orange[3]. Besides, Fenton and Fenton-like methods are commonly used in the degradation of methyl orange as important means[4-7].

Ionic liquids are typical environmental friendly chemicals due to their unique physicochemical properties. In this study, we grafted an ionic liquid monomer onto the surface of the porous silica via chain transfer polymerization, obtaining modified porous silica. A novel catalyst containing palladium was prepared through the adsorption of the modified silica for the metal ion. The catalyst shows remarkable promotion on the degradation of methyl orange in the presence of hydrogen peroxide.

\section{Experimental}

Materials. 1-methylimidazole(99\%), 3-bromopropene(98\%), palladium chloride( $\mathrm{PdCl}_{2}, 59-60 \%$ 
based on Pd), $\gamma$-mercaptopropyltrimethoxysilane(MPTS, 95\%) and sodium silicate(47\% based on $\mathrm{SiO}_{2}$ ) were purchased from Aladdin. Ethyl acetate(AR, $\left.\geq 99.5 \%\right)$,sulfuric acid(AR, 95-98\%), sodium borohydride $\left(\mathrm{NaBH}_{4}, \geq 96.0 \%\right)$, hydrogen peroxide $\left(\mathrm{H}_{2} \mathrm{O}_{2}, \mathrm{AR}, \geq 30 \%\right)$, acetone $(\mathrm{AR}, \geq 99.5 \%)$, $\mathrm{N}, \mathrm{N}-\mathrm{Dimethylformamide}(\mathrm{DMF}, \mathrm{AR}, \geq 99.5 \%$ ) ,methyl orange(IND) and dichloromethane(AR, $\geq 99.5 \%$ ) were purchased from Sinopharm Chemical Reagent Co., Ltd. Toluene(AR, $\geq 99.5 \%$ ) was purchased from Sinopharm Chemical Reagent Co., Ltd and dehydrated with 4A molecular sieve prior to use. 2,2-Azobis (2-methylpropionitrile)(AIBN,AR) was purchased from Sinopharm Chemical Reagent Co., Ltd and recrystallized from acetone prior to use. Other reagents were of analytical grades and used as received.

Characterization. Scanning electron microscope (SEM) measurements were carried out on a FEI(company) Quanta 200F SEM. Nuclear magnetic resonance(NMR) spectra were carried out on a Bruker AVANCE Ш spectrometer. Fourier transform infrared(FT-IR) spectra were recorded on a Bruker TENSOR27 spectrometer using $\mathrm{KBr}$ pellets. Thermogravimetric analysis(TGA) was performed on a NETZSCH STA449F3 thermogravimetric analyzer at a heating rate of $10{ }^{\circ} \mathrm{C} / \mathrm{min}$ under nitrogen atmosphere. Ultraviolet-Visible(UV-Vis) spectra were recorded on a SHIMADZU 2550 spectrometer. The nitrogen physisorption experiment was performed on a QUANTACHROME QUADRASORB SI gas sorption system in static measurement mode at $77 \mathrm{~K}$. The Brunauer- Emmett-Teller(BET) method was applied to determine the specific surface area and pore volume of the porous silica. The Pd loading of the catalyst was determined by an inductively coupled plasma(ICP) spectrometer(Prodigy, Leeman corporation).

Synthesis of Ionic Liquid Monomer. Ionic liquid, 1-allyl-3-methylimidazolium bromide $([\mathrm{Amim}][\mathrm{Br}])$ was synthesized according to a method reported by Zhang et al[8].1-methylimidazole $(6.0 \mathrm{~g}, 73 \mathrm{mmol})$ and 3-bromopropene $(10.0 \mathrm{~g}, 83 \mathrm{mmol})$ were put into a dried flask. The reaction mixture was stirred under $\mathrm{N}_{2}$ at room temperature for $48 \mathrm{~h}$. The mixture was washed with ethyl acetate and dried overnight under vacuum at room temperature. ${ }^{1} \mathrm{H}$ $\operatorname{NMR}\left(400 \mathrm{MHz}, \delta, \mathrm{ppm}, \mathrm{D}_{2} \mathrm{O}\right): 4.10\left(\mathrm{~s}, \quad 3 \mathrm{H}, \quad-\mathrm{N}_{-} \mathrm{CH}_{3}\right), 5.01\left(\mathrm{~d}, 2 \mathrm{H}, J=4.1 \mathrm{~Hz},-\mathrm{N}-\mathrm{CH}_{2}-\right)$, 5.55-5.61(m,2H, $\left.\mathrm{CH}_{2}=\right), \quad 6.18-6.29\left(\mathrm{~m}, 1 \mathrm{H}, \quad \mathrm{CH}_{2}=\mathrm{CH}-\mathrm{CH}_{2}-\right), \quad 7.68-7.70(\mathrm{~m}, 2 \mathrm{H}, \quad-\mathrm{N}-\mathrm{CH}-\mathrm{CH}-\mathrm{N}-)$, 8.98(s,1H, -N-CH-N-).

Preparation of Porous Silica. Porous silica was prepared via sodium silicate hydrolysis. In a typical process, to a solution of sodium silicate $(5.0 \mathrm{~g})$ in deionized water $(50 \mathrm{~mL})$ was added with stirring an aqueous solution of sulfuric acid $(1.8 \mathrm{M}, 20 \mathrm{~mL})$. The reaction mixture was stirred at $75{ }^{\circ} \mathrm{C}$ for $5 \mathrm{~h}$ to form silica gel and then hydrothermally treated at $\mathrm{pH}=10$ at $100{ }^{\circ} \mathrm{C}$ for $24 \mathrm{~h}$. The crude product obtained was washed many times with deionized water and dried under vacuum conditions at $120{ }^{\circ} \mathrm{C}$ for $12 \mathrm{~h}$.

Immobilization of MPTS onto the Porous Silica[9]. Porous silica(1.0 g) was added to a solution of MPTS $(0.2 \mathrm{~mL})$ in anhydrous toluene $(20 \mathrm{~mL})$ and the mixture was stirred under $\mathrm{N}_{2}$ at room temperature for $24 \mathrm{~h}$. The solid was separated from the mixture by filtration and washed many times with dichloromethane and then dried under vacuum conditions at room temperature for $24 \mathrm{~h}$, obtaining MPTS modified porous silica(silica-MPTS).

Preparation of Polymeric Ionic Liquid Modified Porous Silica. Silica-MPTS $(1.0 \mathrm{~g})$ was added to a flask containing a solution of $[\mathrm{Amim}][\mathrm{Br}](1.0 \mathrm{~g}, 4.92 \mathrm{mmol})$ and AIBN $(8.2 \mathrm{mg}, 0.05$ $\mathrm{mmol})$ in $\operatorname{DMF}(20 \mathrm{~mL})$ and the mixture was refluxed under the protection of $\mathrm{N}_{2}$ atmosphere at $80{ }^{\circ} \mathrm{C}$ for $24 \mathrm{~h}$. The solid in the mixture was separated from the reaction system by filtration and then washed many time with dichloromethane. Finally, the product obtained was dried under vacuum at $60{ }^{\circ} \mathrm{C}$ for $20 \mathrm{~h}$, giving polymeric ionic liquid modified silica(silica-PIL). The experimental results show that Silica-PIL can adsorb palladium ions in water, but can not adsorb methyl orange in water.

Preparation of Catalyst. Silica-PIL $(0.1 \mathrm{~g})$ was added into a vessel containing an aqueous solution of palladium chloride $(5.8 \mathrm{mM}, 8.0 \mathrm{~mL})$ and the mixture was sonicated in water bath and then stirred until the solution was discolored. The solid was separated from the mixture by filtration 
and dried under vacuum at $60{ }^{\circ} \mathrm{C}$ for $4 \mathrm{~h}$. To a flask containing the above solid and deionized water $(10 \mathrm{~mL})$ was added with vigorous stirring a cold aqueous solution of sodium borohydride $(0.1$ $\mathrm{M}, 1.0 \mathrm{~mL}$ ) at $0{ }^{\circ} \mathrm{C}$. The product was obtained by filtration and dried under vacuum at $40{ }^{\circ} \mathrm{C}$ for $4 \mathrm{~h}$, affording the catalyst loaded with Pd(Silica-PIL-Pd, Pd: 3.51\%, ICP).

Oxidative Degradation of Methyl Orange by Hydrogen Peroxide in the Absence of the Catalyst. To a vessel containing an aqueous solution of methyl orange $(100 \mathrm{mg} / \mathrm{L}, 3 \mathrm{~mL})$ was added an aqueous solution of $\mathrm{H}_{2} \mathrm{O}_{2}(30 \%, 3 \mathrm{~mL})$. The reaction mixture standed without stirring for $16 \mathrm{~h}$.

Oxidative Degradation of Methyl Orange by Hydrogen Peroxide in the Presence of Silica-PIL. To a vessel containing the mixture of an aqueous solution of methyl orange $(100 \mathrm{mg} / \mathrm{L}$, $3 \mathrm{~mL})$ and Silica-PIL $(0.1 \mathrm{~g})$ was added an aqueous solution of $\mathrm{H}_{2} \mathrm{O}_{2}(30 \%, 3 \mathrm{~mL})$. The reaction mixture standed without stirring for $16 \mathrm{~h}$.

Oxidative Degradation of Methyl Orange by Hydrogen Peroxide in the Presence of the Catalyst. To a vessel containing the mixture of an aqueous solution of methyl orange $(100 \mathrm{mg} / \mathrm{L}, 3$ $\mathrm{mL})$ and the above catalyst $(0.1 \mathrm{~g})$ was added an aqueous solution of $\mathrm{H}_{2} \mathrm{O}_{2}(30 \%, 3 \mathrm{~mL})$. The reaction mixture standed without stirring for $16 \mathrm{~h}$.

\section{Results and Discussion}

Morphology and Physicochemical Properties of Porous Silica. Morphology of the porous silica was studied by SEM observation and shown in Fig. 1. It can be observed that the surface of the sample is scraggly and found many alveolate holes. The special structure may adsorb copious catalytic substances under certain conditions and further provide many active sites for chemical reactions.

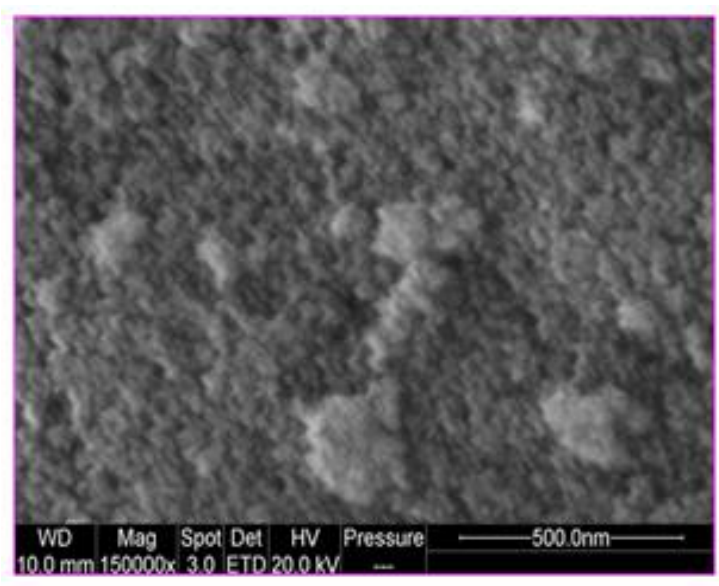

Fig. 1 Morphology of the porous silica

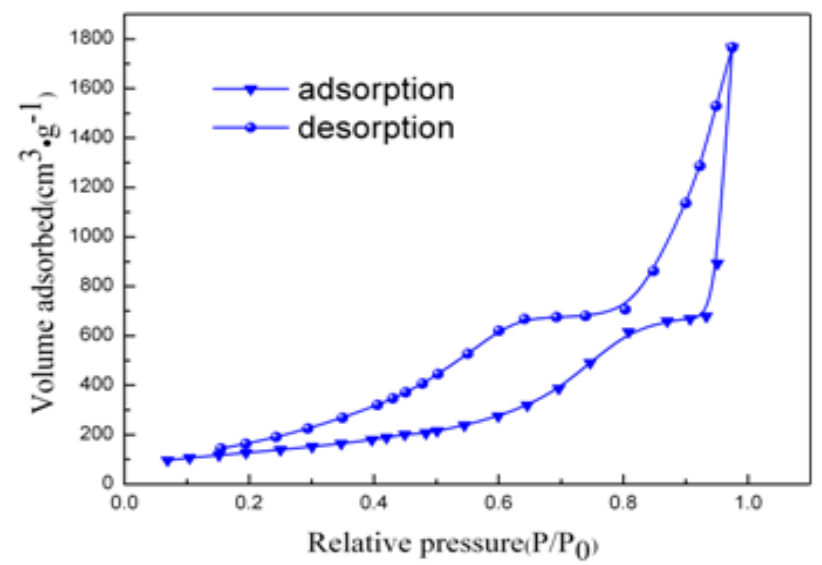

Fig. 2 Nitrogen physisorption isotherm for the porous silica

The specific surface area and pore volume of the porous silica were calculated from the nitrogen physisorption isotherm(Fig. 2). The pore size of the porous silica was determined through the above SEM image. Some parameters on the properties of the porous silica are shown in Table 1.

Table 1 Parameters on the properties of the porous silica

\begin{tabular}{lccc}
\hline & $\begin{array}{c}\text { specific surface area } \\
{\left[\mathrm{m}^{2} / \mathrm{g}\right]}\end{array}$ & $\begin{array}{c}\text { pore volume } \\
{[\mathrm{mL} / \mathrm{g}]}\end{array}$ & $\begin{array}{c}\text { pore size } \\
{[\mathrm{nm}]}\end{array}$ \\
\hline porous silica & 479 & 2.73 & 20.0 \\
\hline
\end{tabular}

Thermal Stability of Silica-PIL. TGA, an effective tool to investigate thermal stabilities of 
various materials, was performed for Silica-PIL from 30 to $850{ }^{\circ} \mathrm{C}$ under nitrogen atmosphere(Fig. 3). For Silica-PIL, the weight loss around $150{ }^{\circ} \mathrm{C}$ is due to the desorption of physic-adsorbed water. In the range of $200-800{ }^{\circ} \mathrm{C}$, a weight loss of $17.37 \%$ is mainly ascribed to the decomposition of organic substances, such as polymeric ionic liquids grafted onto the surface of the sample.

Structural Analysis of Silica-PIL. The structure of ionic liquid-polymer modified porous silica was investigated by means of FTIR spectrum(Fig. 4). The FTIR spectrum manifests a strong band at $1101 \mathrm{~cm}^{-1}$ due to Si-O-Si stretching vibration[10-11]. The bands at 2967 and $2924 \mathrm{~cm}^{-1}$ are ascribed to $\mathrm{C}-\mathrm{H}$ stretching vibration[12]. The spectrum also shows other characteristic absorption bands at 1660 and $1561 \mathrm{~cm}^{-1}$ originated from imidazole ring vibration[13-14].

Evaluation of Catalytic Activities. The color of the aqueous solution of methyl orange oxidated only by hydrogen peroxide was changed from orange to yellow in the absence of the catalyst and kept this color even after 14 days(image (a) in Fig. 5). The result shows that the effect of the degradation of methyl orange only by hydrogen peroxide without any catalyst is poor and the complete degradation of methyl orange in water can not be achieved under the condition. The probable reason is that hydrogen peroxide is stable in the presence of methyl orange[15] and can not produce abundant free radicals to decompose methyl orange. Similar result was obtained for Silica-PIL, suggesting that Silica-PIL has no the catalytic activity for methyl orange.

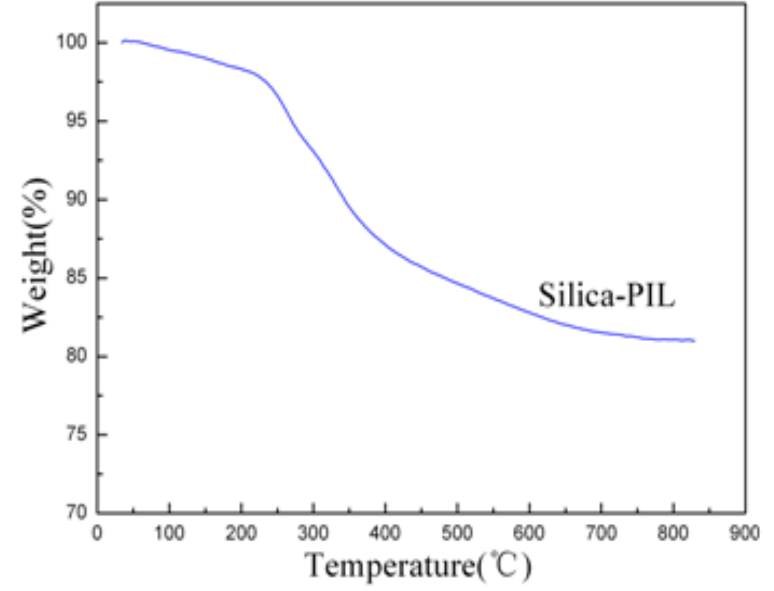

Fig. 3 TGA curve of Silica-PIL

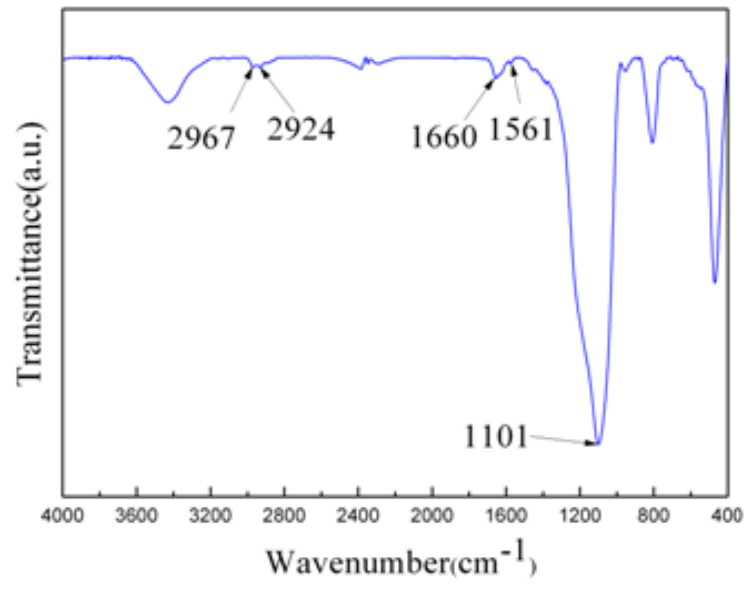

Fig. 4 FT-IR spectrum of Silica-PIL

However, the aqueous solution of methyl orange oxidated by hydrogen peroxide catalyzed by Silica-PIL-Pd was decolorized and became clarified after 16 h(image (b) in Fig. 5), which indicated that the thorough degradation of methyl orange was achieved.

UV-Vis spectra also demonstrated the complete degradation of methyl orange in water oxidated by hydrogen peroxide in the presence of the catalyst(Fig. 6). The band at wavelength of $463 \mathrm{~nm}$ due to the conjugated structure constructed via azo bond[16-17] disappeared after the degradation, indicating that the azo structure of methyl orange molecule was damaged. Benzene ring of methyl orange molecule was also damaged after the degradation owning to the disappearance of band at $273 \mathrm{~nm}$ assigned to $\pi-\pi^{*}$ transition of a benzene molecule[18]. 


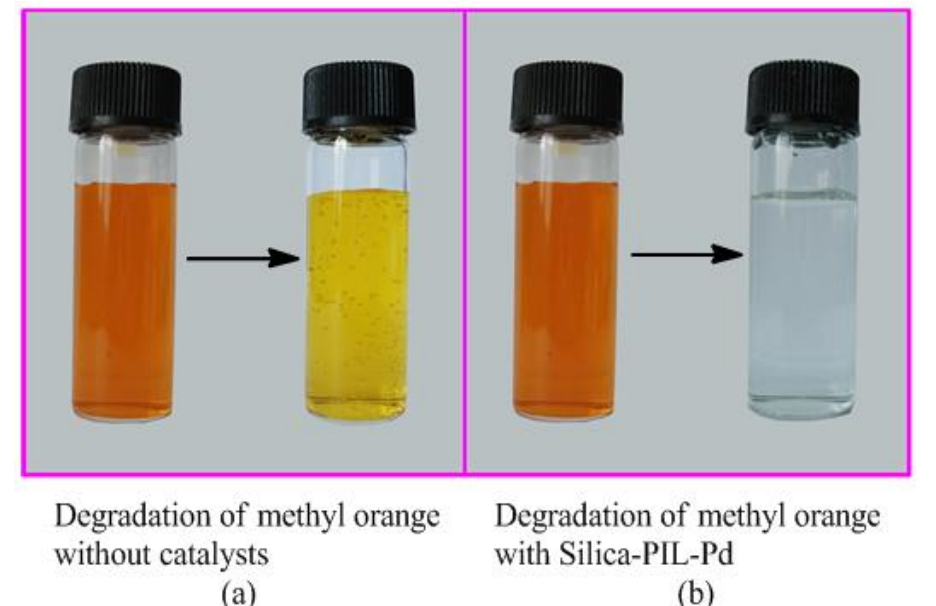

(a)

(b)

Fig. 5 Comparison of the degradation of methyl orange without or with catalyst

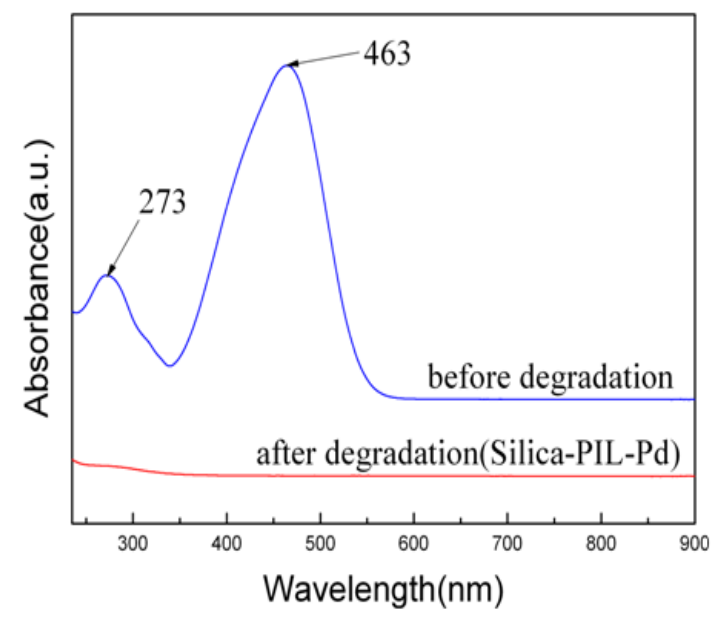

Fig. 6 UV-Vis spectra of methyl orange before/after degradation

to form abundant free radicals, such as hydroxyl radicals[6]. These free radicals can rapidly degrade the methyl orange molecules in water. And the action continued through the diffusion of hydrogen peroxide molecules in the reaction system toward the surface of the catalyst.

\section{Summary}

The synthesis of a novel ionic liquid modified porous silica was achieved via chain transfer polymerization. The hybrid material was used as a kind of catalyst after it was loaded with Pd. The experimental results showed that the degradation of methyl orange catalyzed by the catalyst was significantly accelerated and thoroughly achieved compared with that without the catalyst. The probable reason is that $\mathrm{Pd}$ immobilized on the catalyst facilitates the decomposition of hydrogen peroxide to produce abundant free radicals which can rapidly damage the molecular structure of methyl orange. Our work has significant implications for preparation of novel catalysts and degradation mechanism of azo dyes.

\section{References}

[1] D.N. Thi, H.P. Ngoc, H.D. Manh and T.N. Kim: Journal of Hazardous Materials, Vol. 185 (2011) No.2-3, p.653.

[2] A.H. Xu, X.X. Li, H. Xiong and G.C. Yin: Chemosphere, Vol. 82 (2011) No.8, p.1190.

[3] E. Ember, H.A. Gazzaz, S. Rothbart, R. Puchta and E.R. van: Applied Catalysis B-Environmental, Vol. 95 (2010) No.3-4, p.179.

[4] M.E.M. Ali, T.A. Gad-Allah, E.S. Elmolla and M.I. Badawy: Desalination and Water Treatment, Vol. 52 (2014) No.22-24, p.4538.

[5] A.N. Pham, G.W. Xing, C.J. Miller and T.D. Waite: Journal of Catalysis, Vol. 301 (2013), p.54.

[6] T.Y. Lin and C.H. Wu: Journal of Catalysis, Vol. 232 (2005) No.1, p.117.

[7] V. Shah, P. Verma, P. Stopka, J. Gabriel, P. Baldrian and F. Nerud: Applied Catalysis B-Environmental, Vol. 46 (2003) No.2, p.287.

[8] H. Zhang, J. Wu, J. Zhang and J.S. He: Macromolecules, Vol. 38 (2005) No.20, p.8272.

[9] M. Muellner, J.W. Cui, K.F. Noi, S.T. Gunawan and F. Caruso: Langmuir, Vol. 30 (2014) No.21, p.6286.

[10] F.X. Hu, K.G. Neoh, L. Cen and E.T. Kang: Biomacromolecules, Vol. 7 (2006) No.3, p.809.

[11] C.M. Li, J. Yang, P.Y. Wang, J. Liu and Q.H. Yang: Micorporous and Mesoporous Materials, Vol. 123 (2009) No.1-3, p.228. 
[12] D. H. Williams and L. Fleming: Spectroscopic Methods in Organic Chemistry (World Publishing Corporation, China 2009), p.33.

[13] J.P. Wu, M.J. Wang and J.P.W. Stark: Journal of Quantitative Spectroscopy and Radiative Transfer, Vol. 102 (2006) No. 2, p.228.

[14] N. Nanbu, Y. Sasaki and F. Kitamura: Electrochemistry Communications, Vol. 5 (2003) No.5, p.383.

[15] L. Cheng, M.Y. Wei, L.H. Huang, F. Pan, D.S. Xia, X.X. Li and A.H. Xu: Industral \& Engineering Chemistry Research, Vol. 53 (2014) No.9, p.3478.

[16] Y.P. Chen, S.Y. Liu, H.Q. Yu, H. Yin and Q.R. Li: Chemosphere, Vol. 72 (2008) No.4, p.532.

[17] A.P. Zhang and Y. Fang: Chemical Physics, Vol. 331 (2006) No.1, p.55.

[18] M. Takeuchi, M. Hidaka and M. Anpo: Research on Chemical Intermediates, Vol. 40 (2014) No.6, p.2315. 